\title{
RELIABILITY AND CONDITION MONITORING OF A WIND TURBINE
}

\author{
M. Mohsin Khan, M. Tariq Iqbal, Faisal Khan \\ Faculty of Engineering and Applied Sciences, MUN, St.John's. NL. A1B 3X5
}

\begin{abstract}
Wind is one of the cheapest and cleanest sources of energy. However, large and frequent fluctuations in wind intensity and directions can cause serious problems in harvesting this energy. Wind turbines are subjected to many unexpected environmental loads, which can be catastrophic in nature for the wind turbine system. Like any other industrial equipment wind turbines also require some type of monitoring system which is able to predict the up coming faults of the most sensitive components of the system to save it from a major disaster. This paper highlights the ongoing research on reliability analysis and condition monitoring system required for a small-scale wind turbine system AOC15/50, which is widely used in Atlantic Canada and USA. The paper describes the importance of safety system and lay ground work for sensor specification, sensor mounting and configuration requirements for magnetic tip brake and yaw bearing which were proved to be the least reliable components in an extensive reliability analysis. The paper describes condition monitoring instrumentation, data acquisition system and data analysis methodology.
\end{abstract}

Keywords: Wind turbines, Renewable Energy, Condition Monitoring, Fault prediction, Instrumentation and Measurement.

\section{Introduction}

The need of an independent condition monitoring system has proven to be necessary for many electromechanical systems. Wind turbines installed in remote location need constant monitoring of different variables. Some variables like wind speed and direction, which are primarily important for basic operation are always taken into account, however due to changes in environment other parameters, which under normal situation may not pose any harm, may require attention. Condition monitoring system for wind turbines has been proposed by a number authors [5] and [6]. Most of the large commercial wind turbines have some sort of condition monitoring system but small wind turbines lack this feature due to additional cost involved. With reference to the work [1], an extensive and detailed reliability analysis was carried out for a $50 \mathrm{~kW}$ small wind turbine AOC15/50. That shed some light on the reliability and sensitivity of some of the AOC15/50 components while operating under normal conditions. The result in Table-1 describes the reliability value of wind turbine component discussed in [1]. Tip brake and yaw bearing have been identified as the most venerable components in the analysis and are consistent with the problems faced by other systems in operation.

Table-1: Component Reliability and Failure rate/hr.

\begin{tabular}{|l|l|l|}
\hline \multicolumn{1}{|c|}{ Component } & \multicolumn{1}{|c|}{ Reliability } & Failure rates \\
\hline Tip break & $\mathrm{R}=0.5334$ & $1.00 \times 10^{-4}$ \\
\hline Yaw bearing & $\mathrm{R}=0.9013$ & $0.115 \times 10^{-4}$ \\
\hline Generator & $\mathrm{R}=0.99305$ & $0.769 \times 10^{-6}$ \\
\hline Gearbox & $\mathrm{R}=0.9944$ & $0.63 \times 10^{-6}$ \\
\hline Parking Brakes & $\mathrm{R}=0.999$ & $2.16 \times 10^{-6}$ \\
\hline Blades & $\mathrm{R}=0.9068$ & $\mathbf{1 . 1 1 6 \times 1 0 ^ { - 5 }}$ \\
\hline Blots & $\mathrm{R}=0.9068$ & $\mathbf{1 . 1 1 6 \times 1 0 ^ { - 5 }}$ \\
\hline Hub & $\mathrm{R}=0.9068$ & $\mathbf{1 . 1 1 6 \times 1 0 ^ { - 5 }}$ \\
\hline $\begin{array}{l}\text { Tower and anchor } \\
\text { Bolts }\end{array}$ & $\mathrm{R}=0.9997$ & $\mathbf{1 . 0 0 0 \times 1 0 ^ { - 7 }}$ \\
\hline
\end{tabular}

With advancement in the filed of renewable energy it is deemed necessary to make system more reliable thus achieving higher availability. There is a need for a condition monitoring system to predict the up coming faults and to shutdown the system if necessary. In the following proposed condition monitoring system with all its components is discussed.

\section{Condition Monitoring Parameters}

The next important task after identifying the sensitive components is to specify suitable parameters for monitoring of these components. These parameters will be used to select sensors for collection of data using a data acquisition system and will be used for monitoring. Each component exhibit a unique attribute related to it, which is affected during its use. That attribute can be translated into a measurable parameter like voltage or current, which may vary depending upon different operating conditions. This change will provide information about the operation conditions of different parts of the system. As explained earlier the components proved unreliable need a monitoring system for them. Three parameters selected for these components are brake current, strain and vibration.

\subsection{Current Measurement}

The spoilers or the tip brake are mounted on the tip of each blade that provides aerodynamic drag while stopping. They are a part of regular stopping mechanism and have to be deployed when stop cycle commences. 
However it has been discovered that tip brake being mechanical in nature, have some problems. They are prone to failure during regular operation especially when they are closed. The centrifugal forces acting on them can also lead to a failure. Even in case of magnets activated for keeping them close, brakes seem to open partially or deploy without an instruction from the systems PLC. This type of situation can cause problem towards a smooth operation and energy production. On closed observation it was found that before a complete failure the spoiler would experience mechanical play. This play will introduce some change in the supply current. The change in current is more significant as the break (which essentially is a metal plate connected to spring mechanism and magnet) will vibrate while rotating. Due to the centrifugal force, plates may disconnect from the magnets sending a current surge in the supply as shown in Fig-1. These variations in current can be recorded to keep track of the health of every tip brake. Any sudden variations or a pattern observed by the safety system can be recorded and appropriate action i.e. shutdown the wind turbine may be taken if more then two brakes are vibrating. Vibration induced however current variations, while the system in operation with brakes not deployed will appear in the main power supply to the brakes. The main supply to the brakes is rated at $120 \mathrm{~V} / 5 \mathrm{~A}$. Therefore a current variation of $0-5 \mathrm{Amp}$ is expected. In [2] it is shown the brakes work on a constant AC current value, which implies that any sudden changes in current can be detected.

\section{Fig-1: Expected variation in the brake current.}

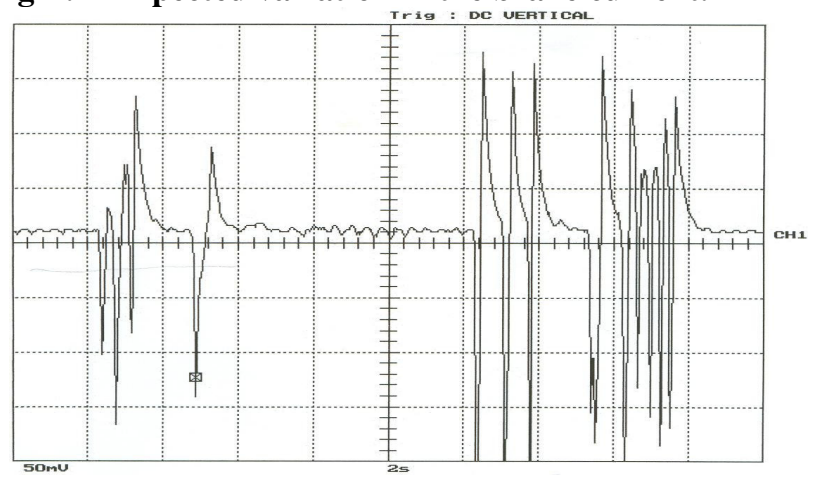

\subsection{Strain Measurement}

Strain measurement is mostly meant for structural loading however it will be serving a slightly different purpose. From analysis in [1] the second most sensitive part of AOC $15 / 50$ appears to be the yaw bearing. It has been observed in similar and other system of larger capacity, that yaw bearing is a prime source to service disruption. That happens in case of bigger yaw bearings, but AOC 15/50 has a yaw bearing not exceeding a diameter of 2 feet. This custom designed bearing under normal operating conditions experiences some unusual moments on it self. Similar bearings have been a cause of failure in many documented cases in other system. The reliability data also suggest the same finding. Being the passive nature of yaw mechanism in $\mathrm{AOC} 15 / 50$, the operating conditions of yaw becomes venerable, as there is nothing to reduce the increased forces in sever weather and wind conditions. Such circumstances may cause some irreversible damage to the bearing's shape, and later on can result in a major system failure. However if such a change is detected before hand, a lot of potential loss can be saved. Before proceeding toward instrumentation the following analysis is deemed necessary. With reference to structural details discussed in [1] and [3], the following will be helpful in designing and calibrating any appropriate sensing arrangement. From Fig 2, which is the reduced scaled drawing provided by the manufacturers and dimensions available in [3] we will proceed as follows;

Fig-2 Drive Train and Yaw support with Tower top

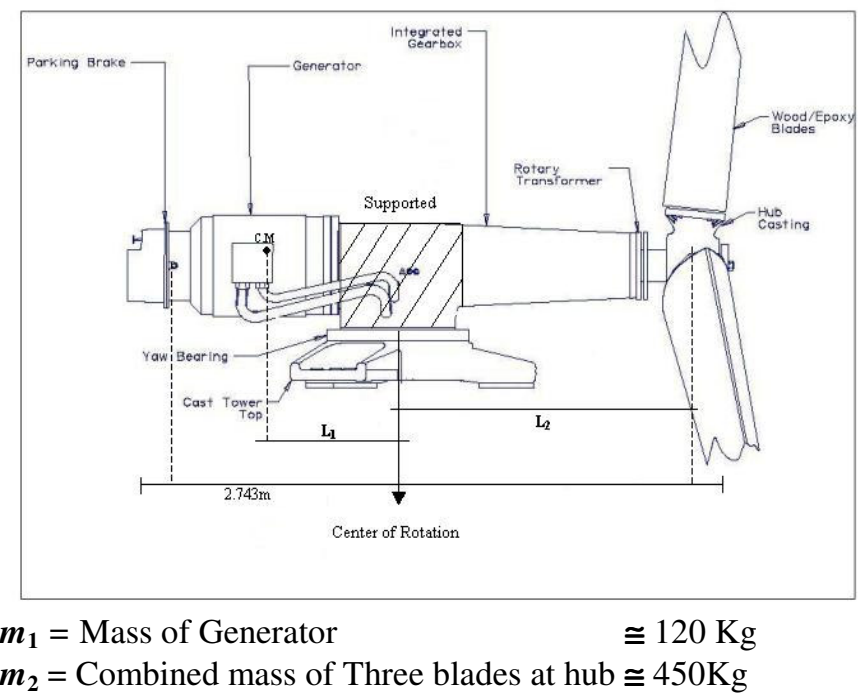

The downward force exerted by both masses as ignoring the mass of gearbox is given by;

$$
\boldsymbol{F}_{\boldsymbol{1}}=1.176 \mathrm{~K} \mathrm{~N} \text { and } \boldsymbol{F}_{\boldsymbol{2}}=4.410 \mathrm{~K} \mathrm{~N}
$$

With moment of force indicated in Fig-2 as $\boldsymbol{L}_{\boldsymbol{l}}$ and $\boldsymbol{L}_{2}$ for both forces respectively the moment of force on the rotating ring of bearing would be;

$$
\boldsymbol{M}_{1}=514.73 \mathrm{Nm} \text { and } \boldsymbol{M}_{2}=4890.48 \mathrm{Nm}
$$

Thus creating an inherent imbalance of moment of about 4375.752 N.m. It is also important to note from Fig-2 that the center of gravity is also slightly towards the Hub rather then being on the center of rotation. This also adds to the imbalance effect on the bearing. Rotor disk thrust phenomenon is discussed in detail in [1]. This thrust can also affect the imbalance conditions. Assuming that while rotating, the blade experience the maximum thrust when they are in a vertically upward position. At the same time the other two blades are at an angle of 120' from it and in a position where they might not be receiving an equal thrust, thus creating a net downward moment on the hub end of the assembly. To keep the analysis conservative a worst-case 
scenario of downward moment due to the difference of thrust to be the total thrust is considered. It means that no force is acting on other blades. From [1] we have the thrust on rotor disk is given as;

$$
\begin{array}{r}
\Delta \mathrm{T}=\mathrm{T}=49.394 \mathrm{k} \cdot \mathrm{N} \\
\mathbf{M}_{\mathrm{T}}=54.775 \times 10^{3} \mathrm{Nm}
\end{array}
$$

Hence the total bending moment on bearing comes out to be;

$$
\boldsymbol{M}_{\boldsymbol{\beta}}=59.1513 \mathrm{~K} \mathrm{Nm}
$$

Which translates into stress $\boldsymbol{\theta}$ of $0.18640 \mathrm{Mpa}$. Using Hook's law to observe change in length per unit length when the above-mentioned stress is applied;

$$
\mathbf{E}=\frac{\varphi}{\varepsilon}
$$

Where $\boldsymbol{\varepsilon}$ strain and $\mathbf{E}$ as young modulus of the material (steel 316), we have a strain value;

$$
\boldsymbol{\varepsilon}=9.65 \times 10^{-7} \mathrm{~m} / \mathrm{m}
$$

After studying the dynamics of bearing which is a 8-point slip bearing designed for heavier loads, the mechanics of transfer of force to the outer bearing ring (mounted to tower) may not be linear, however it is assumed that strain will be transmitted to the outer ring (on which the measuring instrumentation will be done) with same magnitude. For sensitivity and calibration purposes and by convention this value is converted into in/in units and comes out to be;

$$
\boldsymbol{\varepsilon}=0.00003799 \mathrm{inch} / \mathrm{in}
$$

For an available strain gauge with a gauge factor of 2.0 and $\mathrm{R}=120 \Omega$ the relation is given in [4] as;

$$
\begin{aligned}
& \Delta \mathbf{R}=\boldsymbol{\varepsilon} .(\mathbf{G F}) . \mathbf{R} \\
& \Delta \mathbf{R}=0.009118 \Omega \\
& \Delta \mathrm{V} \propto \Delta \mathrm{R}
\end{aligned}
$$

From ohm's law, which implies that there will be a proportional change in the value of voltage with changes in resistance. This provides a basis for a changing parameter for detection.

\subsection{Vibration Measurement}

Vibration measurement has now become a norm in modern condition monitoring systems. The use of such a system is not directed by any analysis most of the time, however to determine the health of rotating machinery a real time vibration measurement technique is required. There are many existing monitoring systems available in markets by different vendors. These systems use Fast Fourier Transform (FFT) routines to compute the FFT and display the magnitude spectrum on the monitor or system display. Most of these systems are custom made and have to be calibrated, moreover they are installed as a software program/application on a Windows/Dos based PC along with a separate data acquisition card that will take the real time data samples and compute the FFT spectrum. These types of systems are suitable for larger systems that have a staggering installation and repair costs. However for smaller systems like AOC15/50 the addition of a separate data acquisition and monitoring system will come out as a huge price issue if one system is to be provided with each wind turbine unit. Keeping in view the financial restrictions there is a need to develop a cost effective and equally efficient solution. An effort is being made during this research to develop such a system that is able to keep track of vibration activity for the whole unit and thus be able to predict sudden changes in frequency spectrum. These changes will be indicative of any up coming faults with in the machine as well as of imbalance with in the frame of turbine that can be a result of yaw movements. FFT is an improved way of computing discrete time Fourier transform (DTFT) which is the crux of the solution. DTFT can be computed on any band limited time varying signal. From its name it is clear that discrete time sample values are required. Mathematical representations of Fourier transform of a continuous time and discrete time signal are given by following equations;

A continuous time signal $\mathrm{x}_{\mathrm{c}}(\mathrm{t})$ can be represented in a discrete time representation as follows;

$$
\begin{aligned}
& x_{c}(\mathrm{t})=x_{k}\left(\mathrm{n} \Delta \mathrm{T}_{\mathrm{s}}\right) \\
& X_{N}(\omega)=\sum_{k=0}^{N-1} x_{k} e^{-j k \omega}
\end{aligned}
$$

Where $T_{s}$ be the sampling time and $X_{n}$ is the DFT of the discrete signal $x_{k}$.

$$
\text { Now } \quad \omega=n \omega_{s} ; \omega_{s}=2 \frac{\pi}{N}
$$

Which gives us the final relation as under;

$$
X_{N}(\mathrm{n} \omega)=\sum_{k=0}^{N-1} x_{k} e^{-j k n \omega s}
$$

This theoretical interpretation of DTFT can be implemented in many ways in code. Fourier transform represented in above equations are known as exponential Fourier transforms, the trigonometric series can also be used to compute the same spectrum. In this particular case Compact Trigonometric Fourier is used in code to compute the Fourier transform the relations used are given as under;

$$
a_{n} \cos n \omega_{s} t+b_{n} \sin n \omega_{s} t=C_{n} \cos \left(n \omega_{s} t+\theta_{n}\right)
$$

The magnitude and Phase spectrum for the above representation given below is used in the code;

$$
\begin{gathered}
\boldsymbol{C}_{\boldsymbol{n}}=\sqrt{a_{n}^{2}+b_{n}^{2}} \\
\boldsymbol{\theta}=\tan ^{-1}\left(\frac{-b_{n}}{a_{n}}\right)
\end{gathered}
$$


Phase spectrum is always given a secondary importance as magnitude spectrum provide enough information in term of harmonics and amplitude of each harmonic, which changes with machine health and upcoming faults.

\section{Instrumentation and Data Acquisition}

The instrumentation required from above discussion is obvious. Instrumentation involves sensors for current, strain and vibration. Typically vibration is measured on rotating machinery by accelerometer, which can be mounted anywhere on the drive train. All three sensors have their own working specifications, so the signals have to be conditioned before the data is recorded and they have to be made compatible with the single board computer. Three signals were conditioned using three different analog circuits for suitable data acquisition. The block diagram for three circuits is as under;

\section{Fig-3 Instrumentation Board Layout}

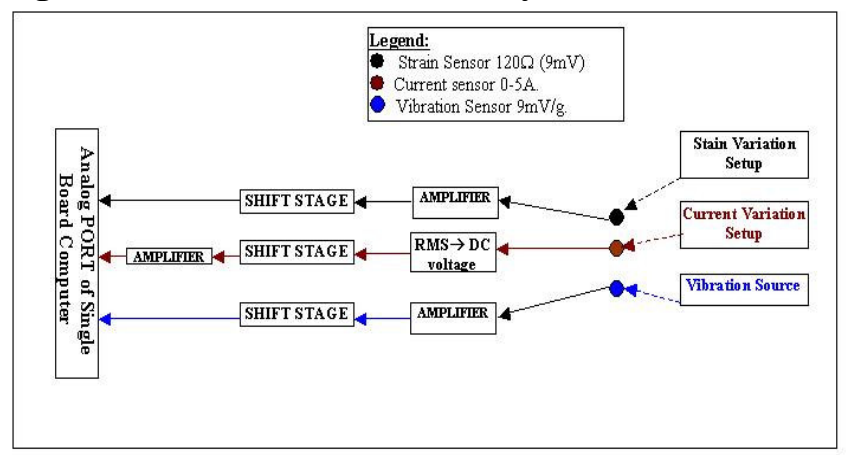

Each signal conditioning circuit is selected after considering the input range of the single board computer (SBC), which is 0-5volts Dc. The SBC is selected keeping in consideration the speed and computation capability required for FFT routines and sampling rates. The processor operates at $40 \mathrm{MHz}$ clock frequency with and $\mathrm{ADC}$ at $66 \mathrm{kHz}$, which is more then what is required. The sampling will be done at slower rate to accommodate the SBC memory issue, as it is only $256 \mathrm{~K}$. Selected SBC (Pico Flash system) comes with a built in DOS utility which makes it easy to operate and userfriendly than other conventional mirco-contorllers. $\mathrm{C}++$ is used to make .exe file for application. This file is loaded into memory and a batch file is programmed in DOS, which include the path of exe file, which runs at startup of SBC. This unorthodox methodology of using SBC has proven to be less problematic and more flexible then conventional methods of data acquisition by micro controllers. A picture of the proposed measurement setup along with the experimental inputs is given in Fig 4.

\section{Conclusions}

This paper describes the ongoing efforts on reliability analysis and development of a condition monitoring system required for a small-scale wind turbine system AOC15/50.
The paper describes the importance of safety system and lays groundwork for sensor specification, sensor mounting and configuration requirements the least reliable components of AOC15/50. The paper describes proposed condition monitoring instrumentation, data acquisition system and data analysis methodology.

\section{Fig-4 Instrumentation Test Bench}

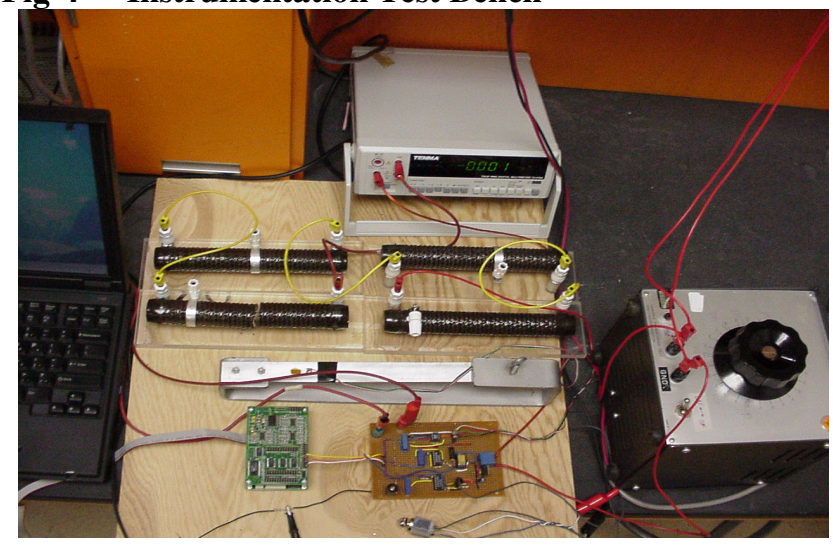

Since the research is still in progress the results of the analysis and performance of the proposed are not included.

\section{Acknowledgements}

The authors would like to thank the Natural Sciences and Engineering Research Council (NSERC), Canada for the financial support towards this research.

\section{References}

[1] M. Mohsin Khan, Tariq Iqbal, Faisal Khan "Reliability analysis of a HAWT” NECEC Proceedings Oct-2004.

[2] Atlantic Orient Corporation Limited "AOC 15/50 Operating and instruction Manual." Revised Versin 5.0 .

[3] Byers and Snyder's “Engineering Mech. Of Deformable bodies"

[4] B.P.Lathi “Modern and Analog Dig. Communication" $3^{\text {rd }}$ Edition.

[5] P. Caselitz, J.Giebhardt " Fault Prediction Techniques for offshore Wind farms maintenance \& repair strategies" ISET, Div of Energy Conversion and Control Engineering. (C) ISET 2003

[6] P. Caselitz, J.Giebhardt "Advanced condition monitoring system for Wind Energy converters" ISET. Proceedings of EWEC'99, Nice, France. 